\title{
Study on the tool setting method of micro- milling tool based on in-line holography
}

\author{
Xianghui Zhang, Jinkai Xu, Zhanjiang Yu, and Huadong Yu* \\ Ministry of Education Key Laboratory for Cross-Scale Micro and Nano Manufacturing, Changchun \\ University of Science and Technology, Changchun, China
}

\begin{abstract}
Aiming at the various shortcomings of existing tool setting methods, this paper proposes a coaxial holographic tool setting method for tiny tools. Based on the research and analysis of the principle of holographic imaging and the key issues of holographic images, a set of holographic tool setting detection device for micro milling tool was built, and the micro milling tool measurement was carried out on the five-axis machining center using standard tools. experiment. Experimental results show that the tool setting device can efficiently perform tool setting detection of micro-milling tool. Compared with the measurement results of the high-precision external presetting instrument, the relative error of the contact tool setting instrument is $0.033 \%$, and the relative error of the holographic tool setting prototype is $0.007 \%$, which is more effective in realizing the tool setting of tiny tools. Detection. This result verifies the feasibility of the coaxial holographic tool setting method for micro tool, that is, holographic measurement can be used for high-precision tool setting of micro milling tool.
\end{abstract}

Keywords: Micro-milling tool, Coaxial holography, Tool setting method.

\section{Introduction}

In the micro-milling process, the diameter and length of the micro-milling tool directly affect the structural size of the workpiece to be machined. Therefore, in order to ensure the dimensional accuracy of the workpiece structure to be machined, it is necessary to obtain the accurate value of the diameter and length of the tool used. Adjust the tool path while compiling the processing program.

High-precision tool setting has always been an important research issue in the field of precision micromachining. Especially with the decreasing size of the preparation of micro tools, the scale range and accuracy requirements of the tool setting are also facing new challenges. The related tool setting methods can be summarized into two measurement methods: contact and non-contact.

In the research of the contact type tool setting method, Ye Kunhuang [1] developed a simple tool setting instrument with a watch. This instrument uses a measuring anvil to carry

\footnotetext{
* Corresponding author: yuhuadong@cust.edu.cn
} 
the tiny force of the tool, and then uses the transmission rod to generate the tiny displacement of the force. The measuring lever passed to the dial indicator. By recording the $\mathrm{Z}$ coordinate value of each tool, and taking the difference between it and the $\mathrm{Z}$ value of the standard tool as its length compensation value; Wei Jiangbo [2,3] developed a mechanical calibration tool setting instrument, which contacts the measuring column through the tool The force generated later causes the inner scale line to coincide with the position of the 0 scale line on the outer scale, so as to obtain the height of the tool setting; when Han Jiahao [4] and others designed a simple photoelectric tool setting instrument, the tool setting The instrument uses high-precision sensors to obtain the tool position information from the tool setting boss and other mechanical transmission parts, and transmits the signal to the CNC system, and finally processes this signal to obtain the $\mathrm{Z}$ coordinate value of the tool. In recent years, the development of contact tool setting instruments has tended to be commercialized. Well-known brands of tool setting instruments at home and abroad include: British Renishaw TS27R tool setting instruments and German Boron Z-NANO tool setting instruments. Various contact tool setting instruments are easy to operate, but for tiny tools with a diameter of $0.5 \mathrm{~mm}$ or less, the tool setting accuracy is low and the tools are easily damaged.

Several foreign companies have advanced experience in the research of non-contact tool setting methods. The Blum tool setting instrument developed by Germany Bolong Technology uses the tool to enter and slowly leave the laser beam range and then processes the signals from the laser transmitter and receiver to complete the measurement of tool parameters. The repeatable measurement accuracy can reach $1 \mu \mathrm{m}$, but the diameter measurement range of the tool is still limited; Zhang Yushi of Changchun University of Science and Technology et al [5] proposed a laser diffraction tool setting method for diamond cutters, and the built tool setting prototype can be The detection accuracy of 0.562 $\mu \mathrm{m}$ is achieved. However, this diffractive tool setting method has not yet been commercialized; the Leader series tool setting instrument developed by Italy M.CONTI company can realize the automatic focus of the part to be measured, and the tool automatic capture and storage of images to determine the position of the tool tip in the measurement coordinate system. The maximum measurement diameter is $320 / 520 \mathrm{~mm}$, and the repeatability measurement accuracy is up to $1 \mu \mathrm{m}$. However, the high-precision presetter relies on imports, is expensive, and tends to be larger Diameter tool measurement.

Coaxial holography is widely used in the detection of object morphology, particle field, biomedicine and other fields due to its advantages such as low coherence requirements and simple recording optical path [6-8]. Based on the research of digital holographic imaging technology, this paper applies holographic imaging technology to the online tool setting detection of micro-diameter milling tool, and proposes a micro-diameter milling tool setting method based on coaxial holography, and explores the high-precision in-position tool setting detection.

\section{Principle of digital coaxial holographic tool setting}

The CNC machine tool automatically executes processing tasks in accordance with the CNC processing program, so before running the program, the tool setting operation must be performed. The key is to make the tool setting point coincide with the tool location point. The tool setting point is generally set on the workpiece or machine tool, and its position can be determined by auxiliary instruments such as high-precision probes. The $\mathrm{X}$ and $\mathrm{Y}$ positions of the tool location point are the same as the tool setting point coordinates. The tool setting technology proposed in this article actually uses holographic imaging to indirectly measure the Z-direction position of the tool position point in the machine tool coordinate system. 
Figure. 1 is a schematic diagram of coaxial holographic tool length measurement. The position $\mathrm{Z} 1$ of the contact end of the machine tool spindle and the tool holder in the machine tool coordinate system is known. Specify a reference position in the image sensor CMOS. The position of the reference in the machine tool coordinate system is: Z2. The position of the main shaft and handle of machine tool in the coordinate system is: Z1. The tool tip reference distance from the image is $\Delta \mathrm{L}$. Then the $\mathrm{Z}$-direction position of the tool is $\mathrm{Z} 1+\mathrm{L}$ ', where the clamping length of the tool is:

$$
L^{\prime}=\left|Z_{2}\right|-\left|Z_{1}\right|-\Delta L
$$

$\triangle L$ can be obtained by collecting the interference pattern recorded by CMOS and using the digital holographic reconstruction algorithm to obtain the holographic reconstruction image of the tool, then suppressing the interference image in the reconstruction result, then extracting the contour of the tool in the reconstruction image, and calculating the distance between the tool tip and the image reference.
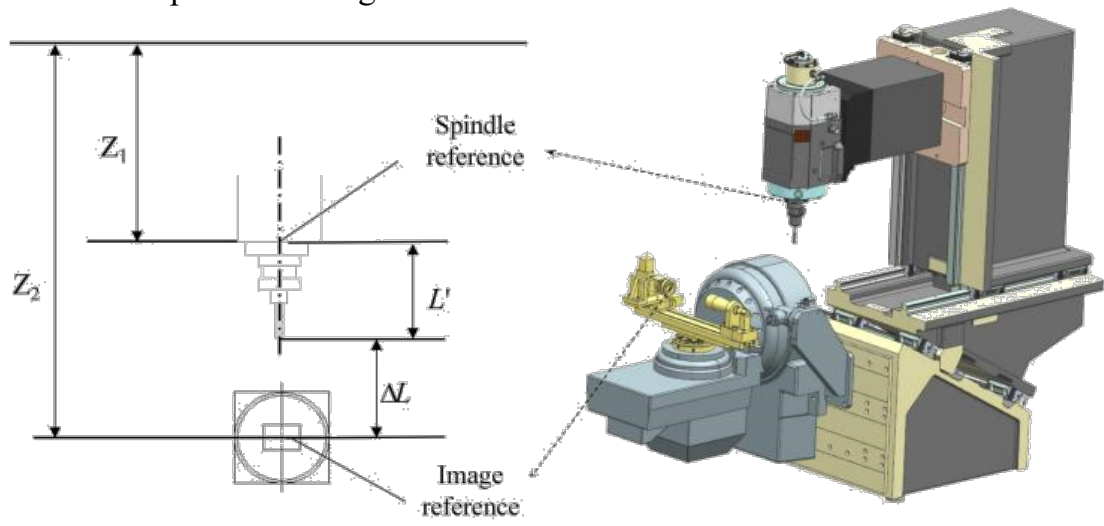

Fig. 1. Schematic diagram of tool length measurement using coaxial holographic.

\section{Tool setting system design and experimental verification}

\subsection{Composition of holographic imaging system}

The structure of the digital holographic tool setting prototype is shown in Figure. 2. The size of the whole machine is $365 \mathrm{~mm} \times 160 \mathrm{~mm} \times 170 \mathrm{~mm}$. The holographic imaging system is mainly composed of lasers, laser beam expanders, miniature industrial cameras, and filters. The components of the imaging system are installed on the base of the machine tool fixture through the $3 \mathrm{R}$ positioning sheet. The profile frame is used to support all imaging devices above and the profile frame is fixed on the $3 \mathrm{R}$ positioning sheet through the profile adapter plate. The design and installation process of the laser mounting frame, the beam expander mounting frame and the camera mounting components must ensure that the laser center, the beam expander center and the CMOS photosensitive surface center are coaxial. The laser mounting frame and the beam expander mounting frame are fixed on the profile frame by screws, and the camera is fixed on a precision sliding table. The $\mathrm{X}$ and $\mathrm{Y}$ directions of the sliding table can be moved freely to adjust the position of the camera's photosensitive surface. 


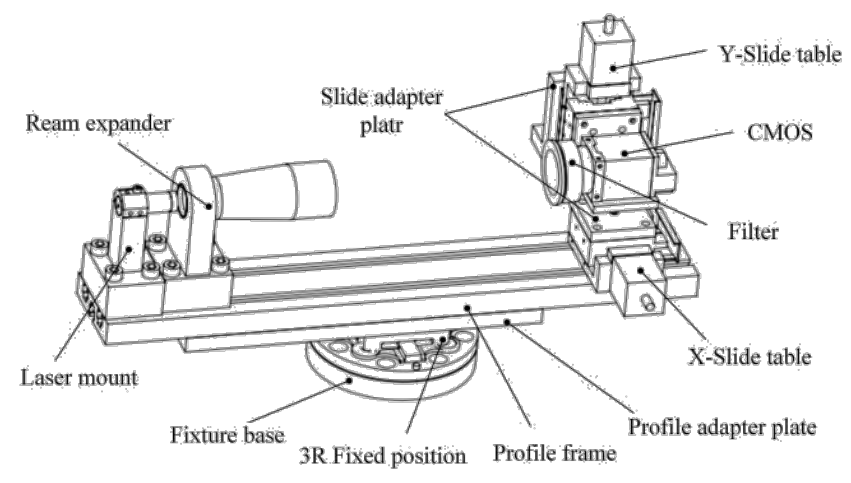

Fig. 2. The structure diagram of digital holographic tool setting device.

\subsection{Experimental verification}

Since the accuracy of the image reference directly affects the measurement accuracy of the subsequent tool length, and in the coaxial holographic imaging, the smaller the size of the object, the more obvious the interference phenomenon, the more object contour information it contains, and the higher the measurement accuracy. The milling tool with a diameter of $0.3 \mathrm{~mm}$ is used as the calibration reference piece, and the reference position of the image is measured and calibrated.

The German Zoller genius3 series is a high-precision tool presetter with a repeat measurement accuracy of $2 \mu \mathrm{m}$. As shown in Figure. 3, the instrument is used to detect milling tool with a diameter of $0.3 \mathrm{~mm}$. The measurement results of the tool clamping length are shown in Table 1.

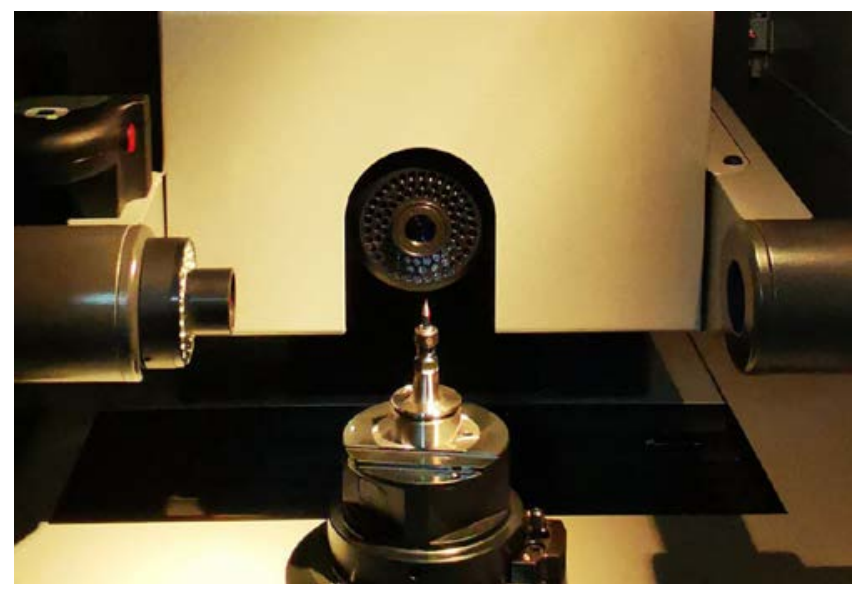

Fig. 3. The process of measuring tool in the Zoller.

Table 1. Zoller Measurement result.

\begin{tabular}{|l|l|l|l|l|}
\hline Number of measurements & 1 & 2 & 3 & Average measured value \\
\hline Clamping length/mm & 77.860 & 77.859 & 77.860 & 77.8597 \\
\hline
\end{tabular}

As shown in Figure. 4, a digital holographic imaging experiment is carried out with a $\Phi 0.3 \mathrm{~mm}$ milling tool. 


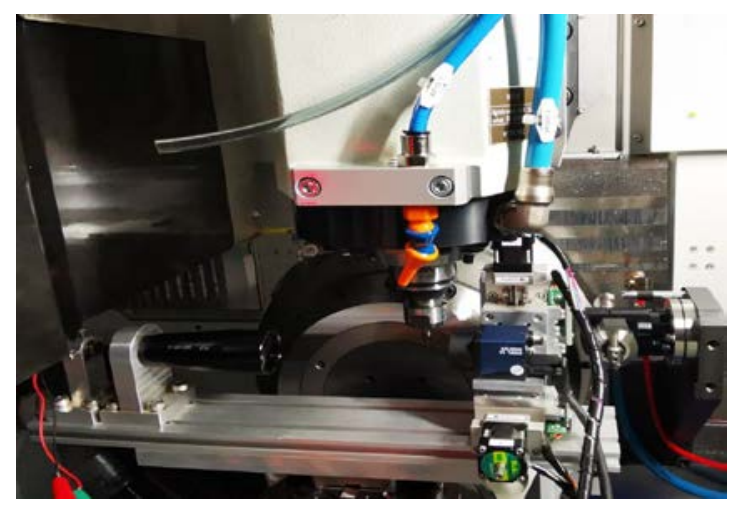

Fig. 4. The process of measuring tool using holographic.

Figure. 5(a) and 5(b) are the digital hologram of a $\Phi 0.3 \mathrm{~mm}$ milling tool and the Fresnel reconstruction image at a reconstruction distance of $30.2 \mathrm{~mm}$ respectively. First flip the image by $180^{\circ}$, and then use the improved self-snake model [9] to perform diffusion filtering on the reconstruction image, and the diffusion result shown in Fig. 5(c) can be obtained. Figure. 5(d) is an enlarged view of the tool contour extracted by the Sobel operator.
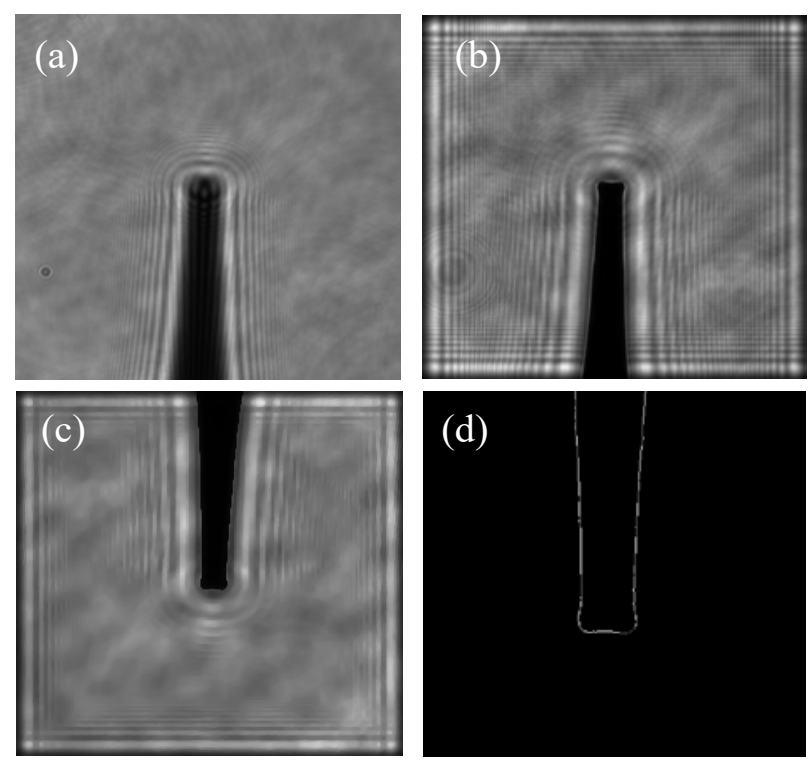

(a)Digital hologram; (b) reconstruction image;

(c) improved self-snake filtering approach; (d) enlarged figure of milling tool contour

Fig. 5. The procedure of measuring the end mill tool with diameter $0.3 \mathrm{~mm}$.

The equations are an exception to the prescribed specifications of this template. You will need to determine By calculating the pixel coordinates of the lower end, it is measured that the distance between the lower end and the center line of the image is 467 pixels, and the corresponding physical distance is $1.6115 \mathrm{~mm}$. The coordinates (unit: $\mathrm{mm}$ ) of the milling tool in the machine tool coordinate system during imaging are: 


$$
\left\{\begin{array}{l}
\mathrm{x}_{0}=-144.4000 \\
\mathrm{y}_{0}=-130.0100 \\
\mathrm{z}_{0}=-188.1350
\end{array}\right.
$$

According to the Zoller measurement results, the average clamping length of the tool is $77.8597 \mathrm{~mm}$, so the position of the image reference (image center) in the machine coordinate system is:

$$
\begin{aligned}
z_{b} & =-\left(\left|z_{0}\right|+\Delta L+L^{\prime}\right) \\
& =-(188.1350+1.6112+77.8597) \mathrm{mm} \\
& =-267.6059 \mathrm{~mm}
\end{aligned}
$$

Figure. 6(a) and 6(b) are the digital hologram of the $\Phi 0.5 \mathrm{~mm}$ milling tool and the Fresnel reconstruction image at a reconstruction distance of $30 \mathrm{~mm}$. First flip the image by $180^{\circ}$, and then diffuse and filter the reconstruction image by improving the self-snake model to obtain the diffusion result shown in Figure. 6(c). Figure. 6(d) is an enlarged view of the tool contour extracted by the Sobel operator. By calculating the pixel coordinates of the lower end, it is measured that the distance between the lower end and the center line of the image is 581 pixels, and the corresponding physical distance is $2.045 \mathrm{~mm}$. The coordinates (unit: $\mathrm{mm}$ ) of the milling tool in the machine tool coordinate system during imaging are:

$$
\left\{\begin{array}{l}
\mathrm{x}_{1}=-144.4000 \\
\mathrm{y}_{1}=-130.0100 \\
\mathrm{z}_{1}=-192.9950
\end{array}\right.
$$

Therefore, the clamping length of the tool is:

$$
\begin{aligned}
L_{0.5}^{\prime} & =\left(\left|z_{b}\right|-\Delta L-z_{1}\right) \\
& =(267.6059-2.0045-192.9950) \mathrm{mm} \\
& =72.6064 \mathrm{~mm}
\end{aligned}
$$
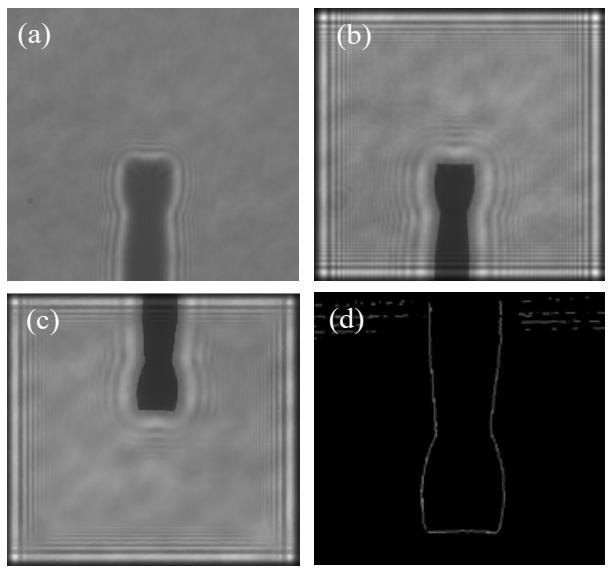

(a) Digital hologram; (b) reconstruction image;

(c) improved self-snake filtering approach; (d) enlarged figure of milling tool contour

Fig. 6. The procedure of measuring the end mill tool with diameter $0.5 \mathrm{~mm}$. 


\subsection{Tool setting accuracy analysis}

In order to compare the accuracy of holographic imaging, as shown in Figure. 7, the tool presetter of the German Zoller genius3 series is used to test the milling tool with a diameter of $0.5 \mathrm{~mm}$. The measurement results of the tool clamping length and diameter are shown in Table 2.

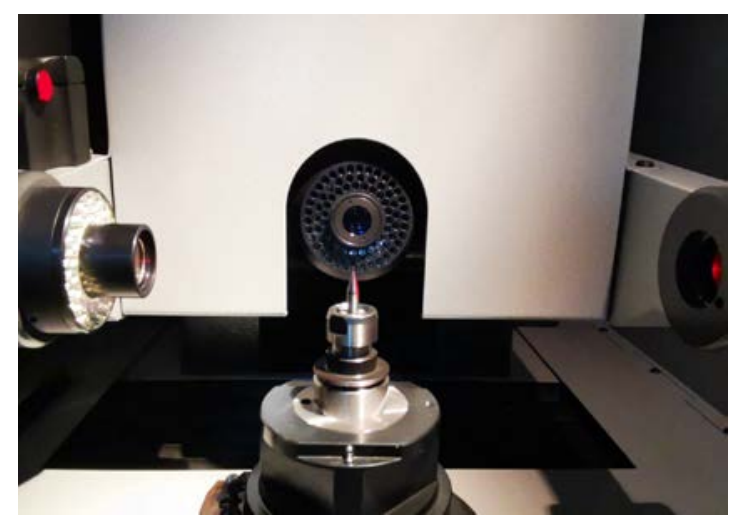

Fig. 7. The process of measuring tool in the Zoller.

Table 2. Zoller Measurement result.

\begin{tabular}{ll}
\hline \multirow{2}{*}{ Number of measurements } & $\Phi 0.5 \mathrm{~mm}$ milling tool \\
\cline { 2 - 2 } & Clamping length/mm \\
\hline 1 & 72.601 \\
2 & 72.601 \\
3 & 72.602 \\
Average measured value & 72.6013 \\
\hline
\end{tabular}

As shown in Figure. 8, the contact type tool setting instrument is used to carry out the tool setting detection of the milling tool with a diameter of $0.5 \mathrm{~mm}$. From the measurement results of the previous tool presetter, the clamping length of the $\Phi 0.5 \mathrm{~mm}$ milling tool is $72.6253 \mathrm{~mm}$.

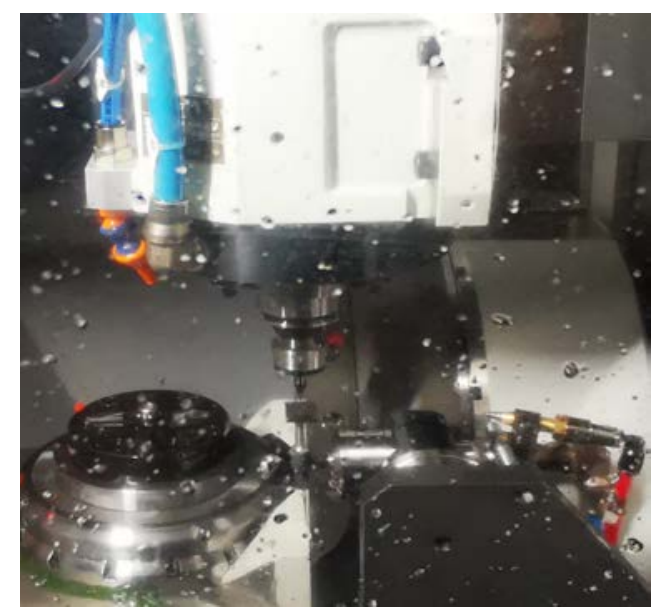

Fig. 8. The experiment of measuring end mill tool, diameter $0.5 \mathrm{~mm}$, using contact tool setting instrument. 
Table 3. Comparison of measurement results of $\Phi 0.5 \mathrm{~mm}$ Milling tool.

\begin{tabular}{|c|c|c|c|}
\hline $\begin{array}{c}\text { Measurement } \\
\text { type }\end{array}$ & $\begin{array}{c}\text { contact type tool setting } \\
\text { instrument }\end{array}$ & $\begin{array}{c}\text { Zoller tool } \\
\text { presetter }\end{array}$ & $\begin{array}{c}\text { Holographic tool } \\
\text { setting device }\end{array}$ \\
\hline $\begin{array}{c}\text { Tool clamping } \\
\text { length/mm }\end{array}$ & 72.6253 & 72.6013 & 72.6064 \\
\hline
\end{tabular}

Table 3 compares the results measured by different methods. For the tool clamping length, compared with the measurement result of the Zoller tool pre-setter, the relative error of the holographic tool setting prototype is $0.007 \%$, while the relative error of the contact tool setting instrument is $0.033 \%$.

$$
\begin{aligned}
& \Delta \mathrm{d}_{1}=\left|\frac{\mathrm{d}_{\mathrm{T}}-\mathrm{d}_{0}}{\mathrm{~d}_{0}}\right|=\left|\frac{72.6064-72.6013}{72.6013}\right| \approx 0.007 \% \\
& \Delta \mathrm{d}_{2}=\left|\frac{\mathrm{d}_{\mathrm{R}}-\mathrm{d}_{0}}{\mathrm{~d}_{0}}\right|=\left|\frac{72.6253-72.6013}{72.6013}\right| \approx 0.033 \%
\end{aligned}
$$

From the above measurement results, it is known that the holographic tool setting prototype can obtain better tool setting detection accuracy than the contact tool setting instrument, and it is more suitable for the tool setting detection of micro-diameter milling tool.

\section{Conclusion}

In this paper, combined with the digital holographic tool setting method of micro-diameter milling tool, the principle and key technology of the tool setting method are discussed. On the existing five-axis high-precision machine tool, the accuracy calibration of the digital holographic tool setting device is completed, and the actual diameter is measured. The 0.5 $\mathrm{mm}$ milling tool is tested for tool setting. The experimental results show that, under the same testing conditions, compared with the measurement results of the high-precision external preset instrument, the relative error of the contact tool setting instrument is $0.033 \%$, while the relative error of the holographic tool setting prototype is $0.007 \%$, which is more suitable used for tool setting inspection of micro-diameter milling tool.

This work is supported by The National Key Research and Development Plan Project (No. 2018YFB1107403); The "111" Project of China (No. D17017); Jilin Province Scientific and Technological Development Program (No. 20190101005JH and No.20180201057GX).

\section{References}

1. K. Ye. "Milling Machine CNC Machining Center based on GSK983Ma Research and Design of the Tool," Equipment Manufacturing Technology, vol.04, pp.45-48, 2015.

2. J. Wei. "Design of mechanical tool checking instrument for NC machine tool," Coastal Enterprises and Science \& Technology, vol.01, pp: 52-54, 2019.

3. J. Wei. "Design of integrated mechanical tool checking instrument for NC machine tool," Die \& Mould Industry," vol.44, pp.55-57+68. 2018.

4. Han, Y. Chen, X. Wang. "A Design of Simple Tools Setting Instrument for CNC Machining Center," Modular Machine Tool \& Automatic Manufacturing Technique, vol.12, pp.99-101+104,2012.

5. G. Shi, Y. Zhang, H. Zhang, J. Wang, Q. Shi, “Analysis of the influence of installation tilt error on the tool setting accuracy by laser diffraction," Applied Optics, vol.57, pp.3012-3020, 2018. 
6. Y. Wang, Y. Xu, X. Jia, G. Lin, T. Liu, Y. Du, X. Meng, "Study on Wear Particle Morphology Detection Based on Digital In-line Holography," Acta Armamentarii, vol.32, pp.1373-1377,2011.

7. L. Yao, X. Wu, X. Lin, Y. Wu, L. Chen, X. Gao, K. Cen, "Measurement of Burning Biomass Particles via High-Speed Digital Holography," Laser \& Optoelectronics Progress, vol.56, 2019.

8. F. Wang, Y. Li, Y. Xie, X. Fan, J. "Digital In-line Holography for Schistosoma Cercariae," Acta Photonica Sinica, pp.92-97, 2012.

9. Y. Cheng, H. Yu, Z. Yu, J. Xu, X. Zhang, "Method of enhancing the quality of in-line holographic images for micro-milling tool,”. Chinese Optics, pp.705-712, 2020.

10. J. Li, M. Wu. Diffraction calculation and digital holography. Beijing, China: Science Press, 2016.

11. C. R. Bramlet. Tool presetting, shrinking and measuring-Zoller, booth 1551 [J]. Modern Machine Shop, 2005(May) 